\title{
Granulomatous Cheilitis Revealing Latent Tuberculous Infection
}

\author{
Georgi Tomov ${ }^{*}$, Parvan Voynov ${ }^{2}$ and Svitlana Bachurska ${ }^{3}$
}

1 Department of Periodontology and Oral Mucosa Diseases, Faculty of Dental Medicine, Medical University of Plovdiv, 15-A “Vasil Aprilov" Blvd. Plovdiv 4002, Bulgaria; georgi.tomov@mu-plovdiv.bg

2 Plastic and reconstructive surgery division, UNI Hospital, 100 Georgi Benkovski Str., Panagyurishte 4500, Bulgaria; ppvoynov@gmail.com

3 Department of Pathology, National Oncology Hospital, 6 Plovdivsko Pole Str., Sofia 1756, Bulgaria; svitba@gmail.com

* Correspondence: georgi.tomov@mu-plovdiv.bg ; Tel.: (+359-896-742-065)

\begin{abstract}
The granulomatous cheilitis (GC) presents a heterogeneous group of disorders characterised by a granulomatous inflammation/reaction of the lips to various stimuli. Numerous etiologies have been proposed, including genetic, immunologic, allergic and infectious. Among the secondary causes of GC, a distant infection by Mycobacterium tuberculosis should be considered. The GC could be the clinical presentation of a tuberculide resulting from a hypersensitivity reaction to an underlying focus of active or latent tuberculosis infection (LTBI). This communication describes a woman diagnosed with GC related to LTBI, who responded well to antituberculosis treatment.
\end{abstract}

Keywords: granulomatous cheilitis, latent tuberculosis, IGRA, antibiotic treatment

\section{Introduction}

Many infectious pathogens may provoke a granulomatous response in the head and neck region and need to be considered a potential aetiology of granulomatous cheilitis (GC) [1]. Among all, Mycobacterium tuberculosis (MBT) is the most common infectious causative agent of granulomatous disease worldwide [2]. The active tuberculosis infection (ATBI) has a more significant burden of MBT than latent tuberculosis infection (LTBI) and acts as an infection reservoir. In contrast, the LTBI is "a state of persistent immune response to stimulation by MBT antigens with no evidence of clinically manifesting ATBI" [3]. An additional aspect of ATBI and LTBI is the tuberculids that are lesions of the skin or mucous membrane resulting from type III or type IV hypersensitivity to mycobacterial antigens disseminated from a distant site of active or latent tuberculosis. For diagnostic purposes is essential to emphasise the fact that MBT cannot be isolated from tuberculid lesions. Tuberculids often develop in patients with silent tuberculosis infection, and few authors consider GC related to ATBI or LTBI as tuberculid due to both lack of detectable MBT and positive response to antituberculosis treatment [4, 5].

This communication aims to present a patient with granulomatous cheilitis diagnosed with LTBI based on a positive interferon-gamma release assay (IGRA) test and treated successfully with isoniazid monotherapy. 


\section{Case presentation}

A 28-year-old female without underlying co-morbidities is presented with progressive upper lip swelling for five months. Other local or systemic symptoms did not accompany it. The primary diagnosis established by the dermatologist was allergic cheilitis (the patient is working in a cosmetic studio), but the swelling did not subside with antihistamines. The local examination revealed a significantly enlarged upper lip with normal color, and slightly tender on palpation. The lip contour and the philtrum were deformed (Figure 1). The nasal mucosa, the floor of the mouth, throat, and tongue were normal. There were no facial nerve palsy or fissured tongue to support MelkerssonRosenthal syndrome. A provisional diagnosis of Miescher granulomatous cheilitis was made.

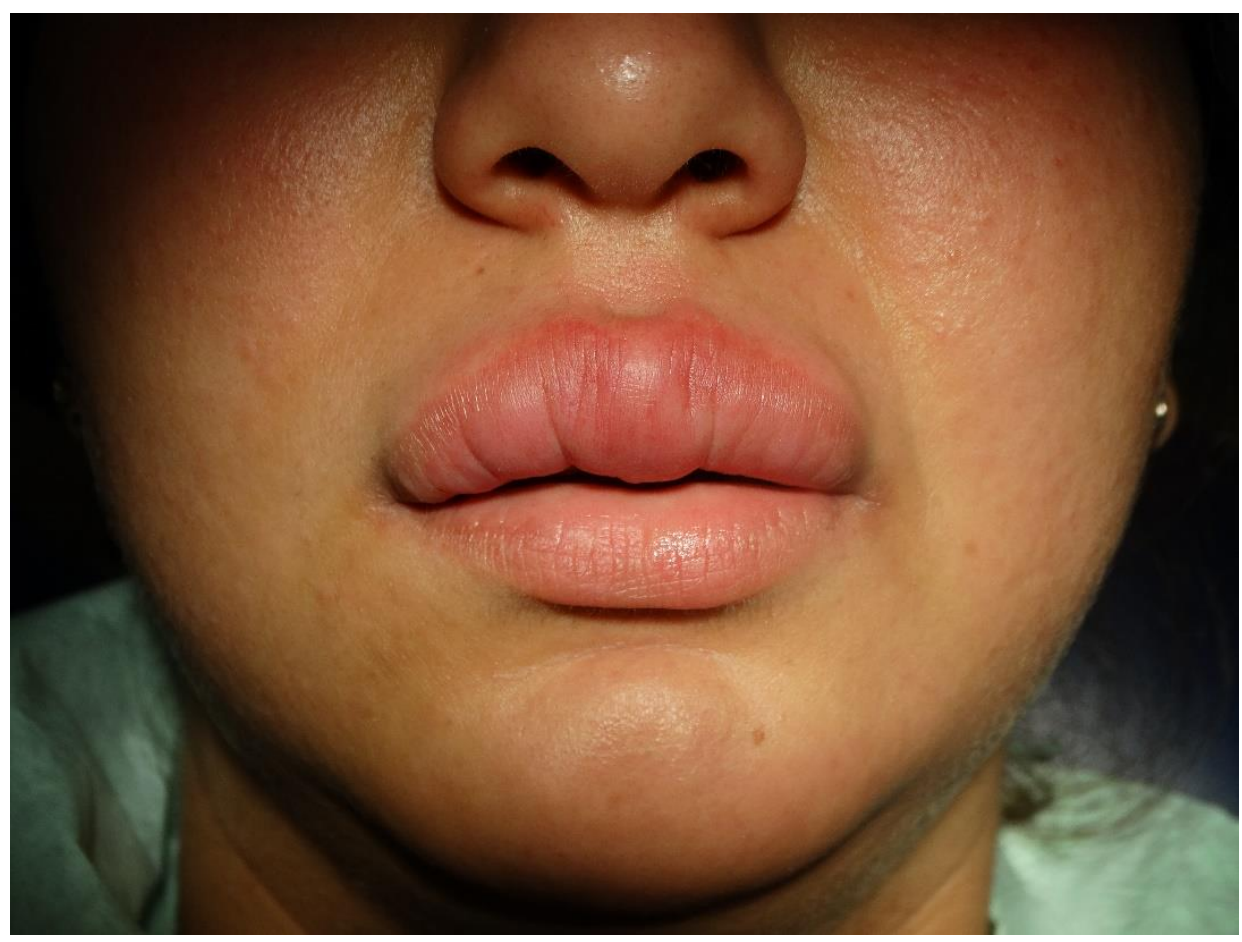

Figure 1. Clinical aspects. Upper lip swelling with blurred and deformed contour.

Additional diagnostic tests were performed to exclude infectious or another origin of the lesion. Laboratory data on haemoglobin, blood cells count, liver and kidney function were within normal limits. The levels of the $\mathrm{C} 1$-inhibitor and $\mathrm{C} 4$ complement factors were normal. The interferon-gamma release assay - IGRA (QuantiFERON-TB gold test) was positive, but the chest radiography was normal (Figure 2). Histopathological examination of the tissue biopsy revealed dilation of lymph vessels and a sparse perivascular lymphoplasmacytic infiltrate admixed with a few histiocytes, some of which tend to form small tuberculoid granulomas. (Figure 3) Both histochemical studies for MBT (Modified Acid-fast Stain) and fungi (PAS) were negative. 

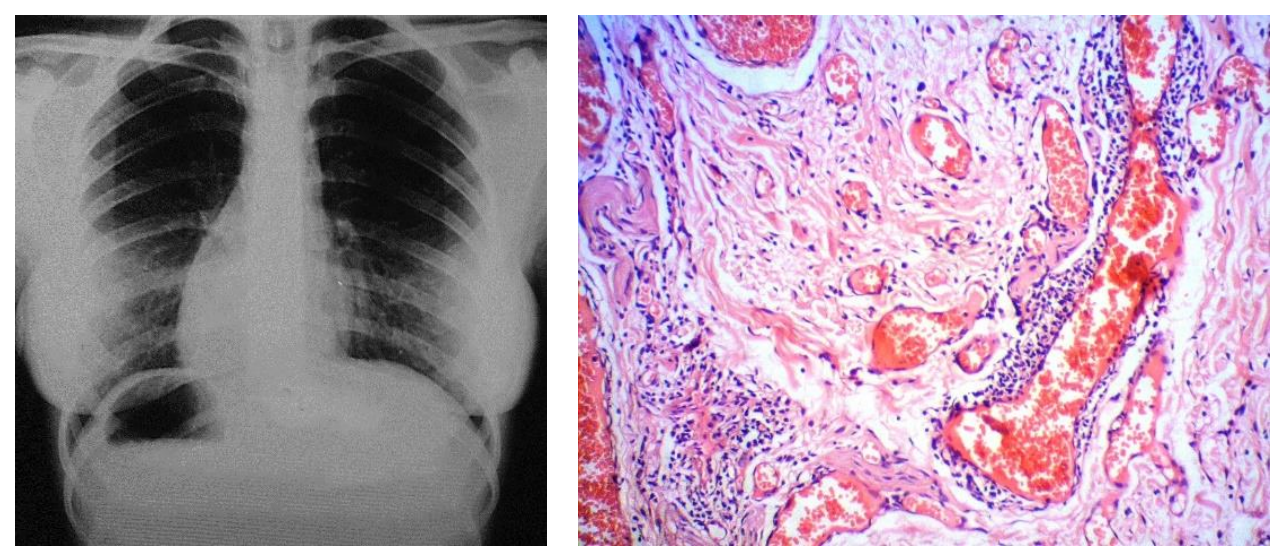

Figure 2. Normal frontal chest X-ray. Lungs and pleural spaces are clear. Cardiomediastinal contour is normal.

Figure 3. Perivascular inflammatory cell infiltrate of lymphocytes and small tuberculoid granulomas (HE, 100x)

The clinical, histological and laboratory findings constellation was sufficient to support a final diagnosis of granulomatous cheilitis related to LTBI. The patient was admitted to a phthisiatry unit and was prescribed monotherapy of Isoniazid (300 mg daily) for six months. After two months the lip swelling started slightly to reduce. After five months, the condition was nearly resolved (Figure 3) but the therapy has continued until a negative IGRA test. One year after treatment the results are stable and the patient is under regular monitoring by an oral pathologist and phthisiatrist.

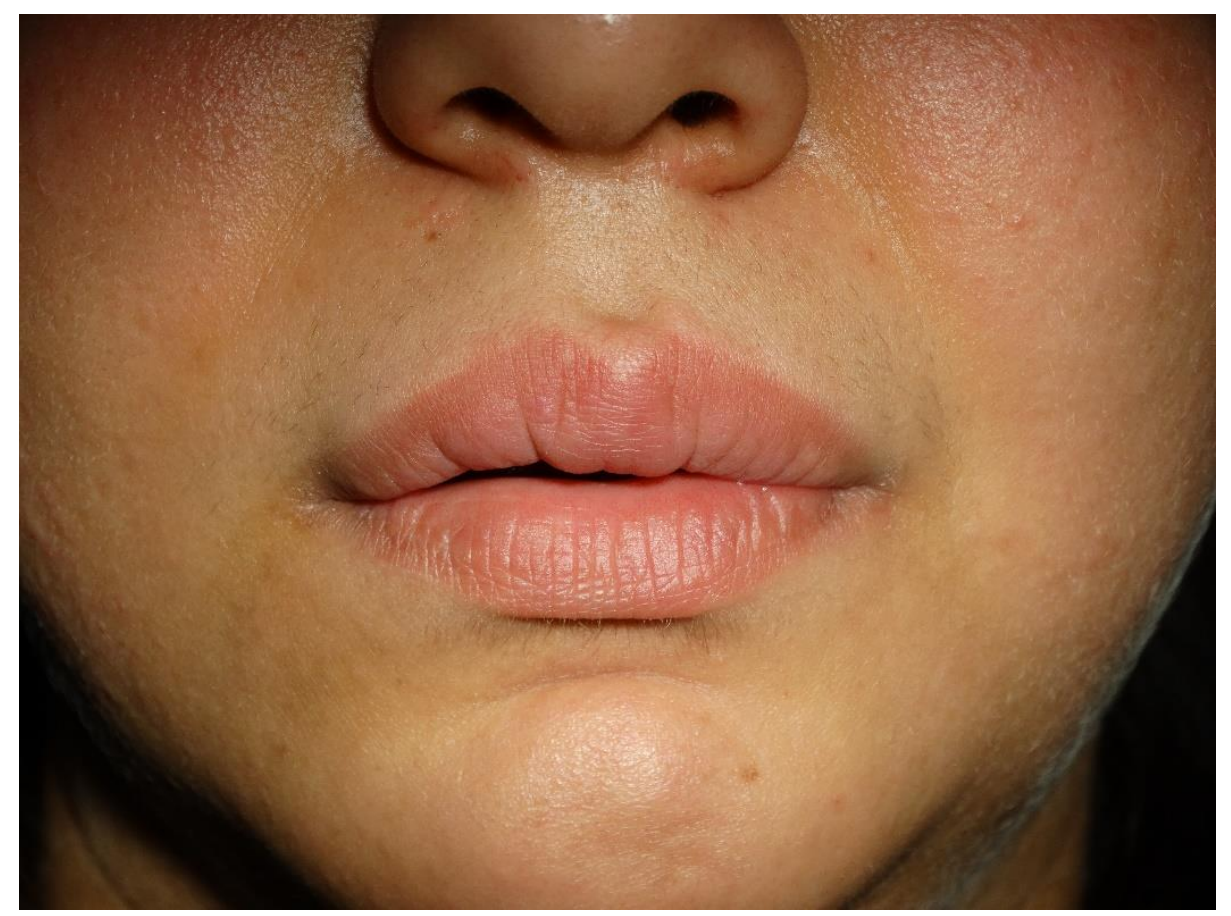

Figure 4. Clinical appearance after five months. 


\section{Discussion}

Although most of the studies excluded MBT as a potential cause of the granulomatous response of the lips, the direct relationship between tuberculosis and GC has been described in a few publications. Kavala M. et al. reported for one case of GC resulting from pulmonary involvement compatible with tuberculosis and improvement following antituberculous chemotherapy for three months [4]. Bhattacharya M. et al. reported a child's GC second of pulmonary tuberculosis [5]. Other two authors described cheilitis as a secondary manifestation of latent pulmonary tuberculosis in adult patients in Cuba [6], respectively in Morocco [7].

In all reported cases, the diagnosis of latent pulmonary tuberculosis is based on microbiological confirmation of MBT (in sputum) and radiography findings. Nevertheless, correct diagnosis of LTBI is also challenging as normal chest radiographs are frequently reported, and different observers often dispute the interpretation of chest radiography results [8]. What is more, the negative mycobacterial culture results do not exclude the possibility of LTBI. Studies emphasised that the spectrum of human tuberculous infection lies within the continuous dynamics of bacterial metabolic activities and hosts' immunological responses [9]. For these reasons, nowadays, we rely much more on molecular detection, and the IGRA test is considered the state-of-the-art method for diagnosing LTBI. In our case, the positive IGRA test and the histological interpretation of the lesion as tuberculide, a hypersensitivity phenomenon not associated with MBT presence, were found to support a diagnosis of granulomatous cheilitis related to LTBI.

Additionally, the positive response to antituberculosis therapy fulfilled the criteria for latent tuberculosis infection manifested with granulomatous cheilitis. In this context, the treatment of the LTBI is also under consideration, especially when the LTBI is displayed with distant lesions. In the reported association cases between GC and LTBI, the preferred treatment modality includes a combination of several anti-tubercular drugs (isoniazid, rifampicin, ethambutol and pyrazinamide) $[4,5,6,7]$. This is in contrast to the recommendations of $\mathrm{WHO}$. According to the guideline, to minimize the risk of acquired drug resistance, treatment for ATBI typically involves four or more antibiotics, while treating LTBI involves only one or two antibiotics [3]. This approach presupposes a low risk of acquired resistance based on the proof for limited replication among the insignificant number of viable bacteria in LTBI [10, 11]. In our clinical case, the recommended monotherapy with isoniazid was applied for six months until negative IGRA.

\section{Conclusions}

Conventional diagnosis methods based on microbiological, histological and radiographical findings may be insufficient to reveal a correlation between subclinical states of TB or LTBI and GC. In summary, we present a young patient with granulomatous cheilitis who was diagnosed and successfully treated for LTBI with isoniazid monotherapy only based on a positive IGRA test. Untreated LTBI may cause chronic tuberculids rarely presented as a recurrent lip lesion. Misdiagnosis (GC of another origin) combined with improper therapy (corticosteroids, antihistamines, etc.) will blur or even deteriorate the clinic. The present report raises concern about the need for more accurate diagnostic and therapeutic guidelines regarding this specific manifestation of LTBI.

Author Contributions: G.T. and P.V. contributed in an evenly manner in literature review and description of the case. P. V. was responsible for the biopsy and S.B. contributed to the histological analysis and visualization. All authors have read and agreed to the published version of the manuscript.

Funding: This research received no external funding

Informed Consent Statement: Written informed consent has been obtained from the patient to publish this paper. 
Acknowledgements: The authors thank Assoc. Prof. Anahid Torossian (The Pulmonology Clinic at University Hospital St. George, MUP) for providing interpretations both on the patient's chest Xray and IGRA test results.

Conflicts of Interest: The authors declare no conflict of interest.

\section{References}

1. Alhassani, A.A.; Al-Zahrani, M.S.; Zawawi, K.H. Granulomatous diseases: Oral manifestations and recommendations. Saudi Dent J. 2020, 5, 219-223. DOI:10.1016/j.sdentj.2019.12.005

2. Fogel, N. Tuberculosis: a disease without boundaries. Tuberculosis (Edinb). 2015, 5, 527-31. DOI: 10.1016/j.tube.2015.05.017

3. World Health Organization. (2018). Latent tuberculosis infection: updated and consolidated guidelines for programmatic management. World Health Organization. https://apps.who.int/iris/handle/10665/260233.

4. Kavala, M.; Südoğan, S.; Can, B.; Sarigül, S. Granulomatous cheilitis resulting from a tuberculide. Int J Dermatol. 2004, 7, 524-7. DOI: 10.1111/j.1365-4632.2004.02211.x. PMID: 15230895.

5. Bhattacharya, M.; Rajeshwari, K.; Sardana, K.; Gupta, P. Granulomatous cheilitis secondary to tuberculosis in a child. J Postgrad Med. 2009, 3, 190-2. DOI: 10.4103/0022-3859.57400. PMID: 19884744.

6. Jorquera, B.E.; Pérez, G.S.; Suárez, M.M.C. Angular cheilitis as an initial clinical sign of tuberculosis. Med Cutan Iber Lat Am 2018, 3, 219-221.

7. Bricha, M.; Slimani, H.; Hammi, S.; Bourkadi, J.E. Chéilite tuberculeuse révélant une tuberculose pulmonaire [Tuberculous cheilitis revealing pulmonary tuberculosis]. Pan Afr Med J. 2016, 24, 176. DOI:10.11604/pamj.2016.24.176.9862

8. Eisenberg, R.L.; Pollock, N.R. Low yield of chest radiography in a large tuberculosis screening program. Radiology. 2010, 3, 998-1004. DOI: 10.1148/radiol.10100485. PMID: 20720079; PMCID: PMC3149157.

9. Delogu, G.; Goletti, D. The spectrum of tuberculosis infection: new perspectives in the era of biologics. J Rheumatol Suppl. 2014, 91, 11-16. DOI: 10.3899/jrheum.140097. PMID: 24788995.

10. Fox, G.J.; Dobler, C.C.; Marais, B.J.; Denholm, J.T. Preventive therapy for latent tuberculosis infection-the promise and the challenges. Int J Infect Dis. 2017, 56, 68-76. DOI: 10.1016/j.ijid.2016.11.006

11. Sterling, T.R.; Njie, G.; Zenner, D.; et al. Guidelines for the Treatment of Latent Tuberculosis Infection: Recommendations from the National Tuberculosis Controllers Association and CDC, 2020. MMWR Recomm Rep 2020, 69, 1-11. DOI: http://dx.doi.org/10.15585/mmwr.rr6901a1 